\title{
AN EXPERIMENTAL STUDY ON THE INTERACTIONS BETWEEN SURFACE WAVES AND FLOATING VISCOELASTIC COVERS
}

\author{
Dharma Sree K. K. ${ }^{1,2}$, Adrian Wing-Keung Law ${ }^{1,2, *}$ and Hayley H. Shen ${ }^{1,3}$ \\ ${ }^{1}$ DHI-NTU Centre, Nanyang Environment and Water Research Institute (NEWRI), Nanyang \\ Technological University, 1 CleanTech Loop, CleanTech One, \#06-08, Singapore 637141 \\ ${ }^{2}$ School of Civil and Environmental Engineering, Nanyang Technological University, N1-01a- \\ 29, 50 Nanyang Avenue, Singapore 639798 \\ ${ }^{3} 132$ Rowley Laboratories, Civil and Environmental Engineering, Clarkson University, Potsdam, \\ NY, USA 13699-5710
}

*Corresponding author: cwklaw@ntu.edu.sg

\begin{abstract}
A possible mathematical ice model for the wave interactions in polar seas was developed based on the assumption that an ice cover behaved as a Voigt viscoelastic material. The dispersion relation was found to depend on the rheological properties of the cover. In the present study, an experimental approach was developed that can enable the verification of the theoretical predictions in the laboratory. The approach utilized the blended mixture of white oil and Polydimethylsiloxane (PDMS) material with various mass percentages of a curing agent, to create a floating layer with a range of targeted viscoelastic properties. Due to the large coverage required for wave flume experiments, special curing procedures were also established for the preparation of PDMS material. The rheological results showed that the mechanical behavior of the floating cover was close to a Voigt material. Experiments were conducted to analyze the wave interactions with the floating viscoelastic cover. The measured data showed an obvious change of wavelength when waves propagated along the cover region. It is observed that the change in wavelength can be linked quantitatively to the viscoelastic properties based on the numerical predictions by Wang and Shen [33]. Some differences were however noted for less viscous covers under longer wave periods. A direct comparison of the PDMS covers with a polypropylene (PP) cover was also performed for verification. Only wave lengthening was
\end{abstract}


observed under the PP cover. With a shear modulus more than three orders of magnitude greater than that of PDMS, the theoretical wavelength for the PP cover from Wang and Shen [33] is very close to that of the thin elastic plate theory from Fox and Squire [7]. Comparison between these two theoretical results and the measured data again deviated with longer wave periods. In both PDMS and PP cases, edge effects and pitching motion of the covers were present at various degrees. In addition, the materials were not strictly a Voigt type. The small deviation from the idealized rheological behavior could also contribute to differences between theoretical and experimental results.

Keywords: ocean waves, floating covers, viscoelastic, model verification, PDMS 


\section{Introduction}

Ocean waves propagating through floating structures has been an active area of research for very large floating structures (VLFSs) [5, 34, 35, 36]. VLFS technology has been developed for floating airports and oil storage bases in Japan, and bridges in the US, Norway, and the United Kingdom. It is in the plan for many more applications including clean energy facilities and space craft launch. There is a large body of literature covering the theories, and existing and potential applications that may be found in the above mentioned references. Most of the VLFSs are for structures that must remain nearly rigid. The negligible deformation supports the assumption of a pure linear elastic response of these structures. However, more flexible structures are sometimes desirable due to the light weight requirement $[10,11,12]$. For future applications of VLFSs, considerations of viscoelastic and more complicated responses to ocean waves may become necessary.

Our present study of wave propagation through viscoelastic floating materials is driven by another important application, namely wave forecasts in ice covered waters. This problem is motivated by the observed rapid decline of sea ice, and the concomitant increase of shipping activities in the Arctic region [26].

Based on the records of National Snow and Ice Data Centre, the areal extent of summer ice in the Arctic is declining at a rate of $-12.9 \pm 1.47 \%$ per decade [29]. The decreasing trend of sea ice extent in the Arctic Ocean is increasing the opportunity of Arctic shipping. This opportunity is not just for ice-capable vessels. With the large amount of open water, it might be possible to travel through the summer Arctic even for open water vessels by 2050 [26]. Thus, Artic shipping can become commonplace in the not so distant future. The study of wave propagation under ice covers is necessary to provide reliable wave forecasts for safer Arctic marine operations. 
Current wave forecast models such as WAVEWATCH III® [30] need a formulation to account for the changes of wave speed and amplitude under different ice conditions. There is a long history of theoretical studies of wave propagation under ice covers, dating back to the work of Greenhill [9]. These theoretical studies are based on different idealizations of an ice cover. One type of idealization considers an ice cover as a distribution of individual elastic thin plates [13, $16,17,20,38,39]$ when the size of plate is comparable to the wave length. The response of finite length individual plate was studied by Meylan and Squire [14, 15]. These plates act as wave scatterers [4]. As waves propagate through them, the energy is redistributed in different directions. Wave attenuation results from the directional redistribution. The other type of idealization considers an ice cover as a continuum that follows certain constitutional law. The properties of the ice cover determine the wave speed and the rate of attenuation. Three classic theories of this type are the mass loading [37], thin elastic plate [31], and viscous fluid [13]. There is extensive development in both types of theories. The most recent review of these theories and related studies can be found in Squire [27].

Ice covers are not uniform homogeneous and isotropic materials. They are conglomerates of a wide range of features depending on the location, time, and history of the ice cover. A partial list of these features includes compression ridges, leads (tensile or shear cracks), rafted level ice, floes (broken pieces of ice), nilas (newly formed thin ice in quiescent water), pancake ice (circular ice disks usually with raised rims), and grease ice (ice slurry formed in turbulence). The glossary and associated images of different types of ice cover may be found at http://aspect.antarctica.gov.au/home/conducting-sea-ice-observations. In the marginal ice zone (MIZ) that borders the open ocean and pack ice, the ice cover may consist of pure pancake/grease ice, or a collection of floes from kilometers down to tens of meters mixed with slushy ice in between. Since most Arctic shipping takes place in the MIZ, where the wave is also the strongest, there is a high interest to find a model that can be applied to the MIZ.

The continuum model is a plausible candidate for different types of ice covers. The three classical types of continuum model mentioned earlier each appears to capture some physical properties of ice covers, with mass loading considering the inertia of the ice floes, thin-elastic 
considering the bending of ice cover, and viscous model considering the energy dissipation in the ice cover. All of these processes are present in the MIZ. Recognizing the coexistence of these processes, a viscoelastic ice cover model was developed by Wang and Shen [33]. This model synthesized the three continuum models: mass loading, thin-elastic, and pure viscous. There are other types of viscoelastic models in the literature [28]. Comparisons of several viscoelastic theories may be found in Mosig et al. [21]. Other types of nonlinear flow rules that are capable of describing energy dissipation have also been proposed [32].

In this study, we investigate the viscoelastic model developed in Wang and Shen [33]. This model was shown to converge to all three types of classical continuum models in proper limits. Mass loading model was obtained by equating complex kinematic viscosity of ice cover to zero whereas elastic plate (with finite thickness) and two-layer viscous model were obtained by substituting shear modulus and viscosity of ice cover to zero respectively. However, results from this pure theoretical work have never been tested experimentally. The main challenge is the difficulty to control and measure the mechanical properties of ice covers. In this paper, we will describe a laboratory study using precisely controlled viscoelastic floating materials to measure the wave celerity. We then compare the results with the theoretical predictions. The same experimental procedures were also applied to test a commercially available and much stiffer polypropylene (PP) cover. In the following sections, we will begin by describing the material preparation, its rheological properties, experimental design of the wave tests, data analysis and results, followed by comparisons with theory, and finally discussions and conclusion.

\section{Viscoelastic material preparation}

To produce precisely controlled floating viscoelastic materials, we developed a technique using an oil-doped Polydimethylsiloxane (PDMS) material, which is widely used in microfluidics for biomedical applications [8]. The density of PDMS material is generally slightly greater than water, hence it needs to be doped with oil to create a floating cover. 
In the present experiments, the main components for the preparation of the oil-doped PDMS viscoelastic floating cover were the prepolymer elastomer base, curing agent, and white oil. The prepolymer (base) and cross linker (curing agent) were available as the commercial two-part elastomer kit, Sylgard 184 (Dow Corning, USA). The white oil, also called paraffin oil, was a colorless, transparent oily liquid soluble in organic solvents. The material properties of these components were described in Prabowo et al. [22] who investigated the standing wave behavior in a small rocking tank with the viscoelastic cover.

The typical preparation of PDMS material involves a curing process whereby the elastomer base is mixed with the curing agent at a particular ratio, and then the material is heat-treated in the oven at high temperatures. Thus, PDMS applications in the literature so far predominantly involved small sample sizes with a length much less than $30 \mathrm{~cm}$ that can be accommodated in a typical oven [22]. To measure the change of wave propagation through a PDMS cover, however, a large sample that can span a few meters in a typical wave flume in the laboratory is needed. Earlier, we had attempted to join smaller oven-cured PDMS pieces to form the large cover [6]. Unfortunately, the joints created strong non-uniformity and localized discontinuity in the mechanical characteristics of the cover. A new curing method was then established in the present study as described below.

The mass percentage of oil and curing agent can be defined respectively as follows:

$$
\begin{aligned}
& m_{o}(\%)=\frac{\mathrm{M}_{\mathrm{o}}}{\mathrm{M}_{\mathrm{o}}+\mathrm{M}_{\mathrm{CA}}+\mathrm{M}_{\mathrm{B}}} \\
& m_{C A}(\%)=\frac{\mathrm{M}_{\mathrm{CA}}}{\mathrm{M}_{\mathrm{CA}}+\mathrm{M}_{\mathrm{B}}}
\end{aligned}
$$

where $\mathrm{M}_{\mathrm{o}}, \mathrm{M}_{\mathrm{CA}}$ and $\mathrm{M}_{\mathrm{B}}$ represent the mass of white oil, curing agent, and base respectively. $m_{o}$, was maintained at $30 \%$ for all the experiments to achieve a target density of $940 \mathrm{~kg} / \mathrm{m}^{3}$. The viscoelastic properties were varied by letting $m_{C A}$ be $4 \%, 6 \%, 8 \%$ and $10 \%$. 
The preparation procedures for the PDMS viscoelastic cover were as follows. After carefully measuring the three components, they were then mixed using an electric mixer (IKA RW 20 digital) for 10 minutes at $500 \mathrm{rpm}$ to ensure a well-mixed solution. Degassing was applied at room temperature $\left(25^{\circ} \mathrm{C}\right)$ inside a vacuum oven for 30 minutes. The mixture was then poured to an aluminum tray with dimensions equal to the size of the cover required for the specific experiment in the wave flume. For the specific PDMS kit used in the present study, the manufacturer had recommended a maximum preparation time of 2 hours at $25^{\circ} \mathrm{C}$ to ensure the proper mixing of the components. Our overall preparation time for the PDMS cover was limited to less than 1.5 hours, thus fulfilling the recommendation.

The prevalent oven-cured approach for PDMS limits the size of the sample as described earlier. To achieve our objective for larger covers, we attempted other approaches for the curing through trials and errors. These included layer-by-layer preparation over large areas with surface heating, and also sequential block-by-block preparation. Finally, we settled on a time-curing approach whereby, the freshly prepared PDMS material was simply allowed to cure for 7 days at room temperature without disturbance. Through rheological testing, it was found that sufficient crosslinking was able to develop under this low-heat long duration curing method. We also found through trials and errors that a long curing period of at least 7 days was essential for the viscoelastic properties to stabilize. Parallel to the larger size PDMS cover, smaller pieces of the identical material and preparation procedure were prepared in rectangular trays $(35 \mathrm{~cm}$ x $25 \mathrm{~cm} \mathrm{x}$ $1 \mathrm{~cm})$ for rheological characterization.

\section{Rheological behavior}

Viscoelastic materials can be modelled using combinations of springs and dashpots [23]. The simplest among the combinations is the Voigt model (parallel combination of spring and dashpot) and Maxwell model (series combination of spring and dashpot). In both cases, the coefficient representing the spring, $G$ and dashpot $\mu$ are constants. Resulting in linear relations between stress and strain/strain rate. The constitutive relation for a Voigt model is given by, 
$\tau=G \gamma+\mu \frac{d \gamma}{d t}$

while that for a Maxwell model is

$\frac{d \gamma}{d t}=\frac{\tau}{\mu}+\frac{1}{G} \frac{d \tau}{d t}$

In the above, $\tau$ is stress, $\gamma$ is deformation, $G$ is elastic modulus and, $\mu$ is dynamic viscosity. To measure the properties of a viscoelastic material in a rheometer directly, a torsional test with oscillatory shearing is commonly used. For an oscillatory shear test, the material is subject to an angular frequency $\omega$ and a given maximum strain, whereby the resistance torque is measured to calculate the storage modulus $G^{\prime}$ and loss modulus $G^{\prime \prime}$. These moduli are related to the elastic modulus and dynamic viscosity. For a Voigt material, the relationships are

$G^{\prime}=G$, a constant; $\quad G^{\prime \prime}=\omega \mu$

and for a Maxwell material, the relationships are

$G^{\prime}=\frac{G \xi^{2} \omega^{2}}{1+\xi^{2} \omega^{2}} ; \quad G^{\prime \prime}=\frac{G \xi \omega}{1+\xi^{2} \omega^{2}}$.

In the above $\xi=\mu / G$ is the relaxation time. Thus during an oscillatory rheometer test, for a Voigt model, $G^{\prime}$ remains constant when a frequency sweep from low to high $\omega$ is applied, while $G$ " increases linearly with $\omega$ resulting in a constant dynamic viscosity $\mu$. The Maxwell model is however very different, with $G^{\prime}$ increasing with $\omega$ and approaching an asymptote when $\omega>1 / \xi$, while $G$ " increases with $\omega$ to a maximum at $\omega=1 / \xi$ then decreases.

The viscoelastic properties of oil doped PDMS were measured using a commercial rheometer (Anton Paar, MCR 302, Germany, with a parallel plate geometry of $20 \mathrm{~mm}$ diameter). The test samples were cut out of the smaller PDMS pieces using circular punch of diameter $20 \mathrm{~mm}$. Our measurements were done with $1.6 \mathrm{~mm}$ thickness based on the required specifications [23].

Amplitude sweep was first performed in the rheometer tests to determine the linear viscoelastic range. The viscoelastic properties of the PDMS material were then analyzed using a frequency 
sweep analysis from 0.01 to $100 \mathrm{rad} / \mathrm{s}$ with a constant strain amplitude of $0.10 \%$, which was within the linear range. Three samples were tested for each $m_{C A}$ to ensure the repeatability of the test results which are shown in Figure 1. From the figure, the rheological behavior of the PDMS material closely resembled the Voigt model with $G^{\prime}$ almost independent of rotational frequency and $G^{\prime \prime}$ varying with rotational frequency nearly linearly. Note that both $G^{\prime}$ and $G^{\prime \prime}$ increased with $m_{C A}$. Figure 1 also shows that $G$ " was slightly nonlinear with a gradual increase over four orders of magnitude in the shear frequency. The theoretical model by Wang and Shen [33] used the Voight model to define the viscoelastic cover, hence our PDMS material is suitable for testing this viscoelastic theory. The evaluation of dynamic viscosity from the loss modulus will be discussed in Section 6.1.

For the wave periods considered in the present experiments, the viscoelastic floating covers had larger values of $G^{\prime}$ compared to $G^{\prime \prime}$ (see Table 1), and the ratio of $G^{\prime \prime} / G^{\prime}$ decreased with increasing $m_{C A}$. From $m_{C A}=4 \%$ to $10 \%, G^{\prime \prime} / G^{\prime}$ dropped by a factor of 2 , i.e. the material became relatively more elastic than viscous. Finally, we measured the rheological behavior of the PDMS material over a three-week duration after curing, and found that it remained stable. Thus, the wave experiments could be performed assuming that the viscoelastic properties remained unchanged within this duration.

We performed experiments with the PDMS as well as commercially available polypropylene (PP) covers for comparison. Both types of covers had the same thickness of $0.01 \mathrm{~m}$. The properties of the PP cover were determined using dynamic mechanical analysis (DMA Q800 V20.9 Build 27) to be $G^{\prime}=870 \mathrm{MPa}$ and $G^{\prime \prime}=24 \mathrm{MPa}$, and the density was $910 \mathrm{~kg} / \mathrm{m}^{3}$. As a viscoelastic material, $\mathrm{PP}$ is thus much stiffer than the PDMS with $m_{C A}=10 \%$. The storage modulus of the PP cover was nearly constant and so was the loss modulus over the entire range of frequency test from 0.1 to $100 \mathrm{~Hz}$. Hence, the PP cover was strictly speaking not a Voigt type or a Maxwell type material.

\section{Experimental setup}


The experiments were conducted in a wave flume made of tempered transparent glass panels supported with a stainless steel framework. The wave flume was $8 \mathrm{~m}$ long, $0.3 \mathrm{~m}$ wide and $1.0 \mathrm{~m}$ deep. It was equipped with a piston-type wave generator (see Figure 2) that can generate regular waves with a wide range of wave periods. Mesh type wave absorbers were installed at both ends of the flume. The vertical upstream absorber filtered energy of the standing wave formed behind the wave generator. The downstream absorber had a slope of 1:1.25 and it acted as the dissipation beach to minimize the reflected wave energy.

The surge as well as the drift motion of the floating cover was prevented by a vertical stopper rod at the trailing edge of the cover. The stopper rod was kept in position by tightening to the steel framework of tank and with its square base resting on the bottom of tank. It should be noted that in the literature, various methods had been employed to constrain surge. The experimental study on floating plates by Sendil and Graf [24] used mooring lines attached to the floor to keep the plate in position. In the laboratory experiments conducted by Bennetts et al. [2], loose moorings attached to the side walls were used. The wave basin experiments conducted by Montiel et al. [18] for the hydroelastic analysis of floating disks used two vertical rods (one passed through a hole at the center of the disk and the other was close to the edge) to restrict horizontal motion (surge and sway) as well as rotations (yaw). Both rods acted like vertical cantilevers with their top end fixed to a platform above. The influence of the center rod on flexure modes was found to be insignificant [19]. Despite the different approaches, the stopper rod used in the present experiments does not have any physical attachment with the cover, hence its effects on the vertical displacement and deformation of the floating cover was assumed to be negligible.

The experimental conditions included a range of monochromatic waves with periods from $\mathrm{T}=$ 0.5 to $1.0 \mathrm{~s}$, while the water depth was held constant at $0.30 \mathrm{~m}$. The stroke of the wave generator was controlled by a capacitance wave gauge to produce an incident wave of constant amplitude. The repeatability of the wave generation was checked to be excellent. Wave conditions generated by the same setting always produced nearly identical time series (see Figure 3 ). The wave period 
range was selected such that the reflection from the absorbing beach was small and also the waves were not too steep. The optimum wave amplitude $a=0.0085 \mathrm{~m}$ was chosen because smaller amplitudes would cause larger errors in the analysis, while larger amplitudes would lead to significant nonlinear effects and wave breaking. Table 2 provides the values of wave steepness used, in which the open water wave number $k_{0}$ and the wave steepness $k_{0} a$ are provided.

Ultrasound sensors were installed above the floating cover to monitor the surface displacement during the passage of surface waves. The ultrasound sensors were synchronized by a common data acquisition system (NI 9215, National Instruments), and the time series data were recorded using LABVIEW. A total of three ultrasound sensors (US325, General Acoustics) were used with resolution of $0.18 \mathrm{~mm}$ and sampling frequency of $50 \mathrm{~Hz}$ (Ultra lab 40D, General Acoustics). Vertically, they were held at a distance of $5 \mathrm{~cm}$ above the cover as the blind area for the sensor was $3 \mathrm{~cm}$. With the $1.0 \mathrm{~m}$ PDMS and PP covers, the position of the first ultrasound sensor (US1) was fixed at $10 \mathrm{~cm}$ from the leading edge. The two other ultrasound sensors were placed at $10 \mathrm{~cm}$ and $50 \mathrm{~cm}$ from US1 respectively, in the first set of experiments conducted for different wave periods. Each experiment was performed at least three times to check for repeatability. Subsequently, the two sensors were moved to $20 / 60 \mathrm{~cm}, 30 / 70 \mathrm{~cm}$ and $40 / 80 \mathrm{~cm}$ from US1 in the second, third and fourth sets of experiments, respectively. In each of these subsequent sets of experiments, the wave conditions were identical. In this manner, the time series of surface displacement data at 9 locations of the cover were obtained with a total of 72 experiments for each test condition (i.e. 6 wave periods $\times 4$ position changes $\times 3$ repetitions). With the longer $2.45 \mathrm{~m}$ PP cover, US1 was fixed at $75 \mathrm{~cm}$ from the leading edge instead, while US2 and US3 were shifted by $20 \mathrm{~cm}$ after each set of experiment. Thus, the time series of surface displacement data at 7 locations were obtained $(6$ wave periods $\times 3$ position changes $\times 3$ repetitions).

Overwash was always observed at the edges of PP covers in initial trial experiments conducted. Both $G^{\prime}$ and $G^{\prime \prime}$ of the PP cover were very large compared to the PDMS covers, which restricted its deformation with the wave. Skene et al [25] developed a theoretical model for overwash with a thin elastic plate model. The model predictions were validated by laboratory experiments 
using PP and PVC sheets. To prevent the overwash, we used a $1 \mathrm{~cm}$ thick Styrofoam edge barrier attached to the leading and trailing edges of the PP cover for the subsequent experiments. All results reported here for the $1.0 \mathrm{~m}$ and $2.45 \mathrm{~m}$ long PP covers had the barriers. A similar approach was used before for the experiments conducted by Montiel et al, [18] using neoprene foam edge barrier. Because of this modification, the wave characteristics near the edges of the cover might be influenced.

\section{Experimental Results}

\subsection{Wave celerity along viscoelastic covers}

The data rate was first increased from $50 \mathrm{~Hz}$ to $200 \mathrm{~Hz}$ by numerical interpolation using the PCHIP function (Piecewise Cubic Hermite Interpolating Polynomial) in MATLAB to enable more precise determination of the wave crests. Since the trailing edge of the $1 \mathrm{~m}$ long cover in the experiments was positioned at $2.90 \mathrm{~m}$ from the end wall of the wave flume, the minimum time required for the longest wave $(T=1.0 \mathrm{~s})$ to reach the cover after reflection from the end of the flume was approximately 4 seconds. Hence, the first three waves which were judged to be fully developed in the wave record free from reflection were considered in the analysis as shown in Figure 3.

The wave celerity was calculated as

$$
c_{s}=\frac{\Delta x_{i}}{\Delta t}
$$

where $\Delta x_{i}$ is the distance of the $i^{\text {th }}$ ultrasound sensor from US1, and $\Delta t$ is the corresponding time lapse for the crest to travel. The average celerity for the three waves considered was calculated for each position of ultrasound sensor. The error plot for the celerity was drawn using errorbar $(Y, E)$ function of MATLAB where $Y$ is the mean of the 3 repetitions and $E$ is the corresponding standard deviation. Figure 4 shows the measured $c_{s}$ along the viscoelastic cover 
region which was clearly different from the open water celerity, $c$. The difference was also highly dependent on both the material and incident wave characteristics. For longer wave periods, $\mathrm{T}=0.7$ and $1.0 \mathrm{~s}, c_{s}$ was smaller than $c$ as shown in Figures $4 \mathrm{a}$ and $4 \mathrm{~b}$ which implied wavelength shortening in the dispersion relationship. However, for the shortest wave period tested, $\mathrm{T}=0.5 \mathrm{~s}, c_{s}$ became larger than $c$ for the more elastic covers (with larger $m_{C A}$ ) as shown in Figure 4c. For all the wave periods, $c_{s}$ generally increased with shear modulus.

The determination of wave celerity involved the examination of the wave profile at different locations. We also attempted to determine the reduction of wave magnitude, or wave attenuation, along the cover at the same time. However, it was found that the cover length was too short for a reliable attenuation measurement.

\subsection{Spectral Analysis}

Figure 5 shows the spectral analysis of the surface displacement at different locations along the covers based on the ultrasound sensor data for the PDMS viscoelastic covers as well as the $1.0 \mathrm{~m}$ long PP cover. The discrete Fourier transform $(|Y(\sigma)|)$ of the time series data was calculated using $f f t$ () function of MATLAB. As discussed previously, only the first three fully developed waves were considered.

With the PDMS covers (see Figure 5a), the wave spectrum at different locations varied slightly. Comparatively, the wave spectrum for the $1.0 \mathrm{~m}$ PP cover shown in Figure $5 \mathrm{~b}$ had obvious larger peaks towards the two free edges of the cover and smaller peaks in the middle. Using a thinelastic plate theory, Fox and Squire [7] and Andrianov [1] as well as many other studies found that due to the existence of evanescent and two damped travelling waves at the edges of an elastic plate, the vertical displacement near the edges was larger than the middle section of the plate. In addition, for waves that were longer than the floating plate, significant pitching might occur $[3,15]$. Both phenomena were possible sources of the observed non-uniform spectral 
energy along the cover. Theoretical study of deflecting and pitching of a finite size viscoelastic cover over water waves, to the authors' knowledge, has not been done.

Figure 6 provides the spectral analysis results for the $2.45 \mathrm{~m} \mathrm{PP}$ cover. As mentioned earlier, to prevent overwash, Styrofoam barriers were installed on the leading the trailing edges. Two cases are shown, one with $\mathrm{T}=0.7 \mathrm{~s}$ and the other with $\mathrm{T}=0.5 \mathrm{~s}$. Comparing Figure 5 with Figure $6 \mathrm{a}$, both for $\mathrm{T}=0.7 \mathrm{~s}$, it is seen that the variation of peaks of the spectra is reduced significantly with a longer cover size. Comparing Figure $6 a$ with $6 b$, it is observed that the finite size effects were slightly diminished when the wavelength reduces. The effect of finite length of cover on the celerity and wave number measurement is discussed in Section 6.3.

5.3. Wave celerity along $2.45 \mathrm{~m}$ polypropylene cover

The results of the average wave celerity along the $2.45 \mathrm{~m}$ long PP cover are shown in Figure 7. The recorded wave celerity was higher than the open water celerity, implying that wave lengthening occurred for all wave period cases. The average celerity for the first two locations closer to US1 was typically faster. With increasing distance from the leading edge, the average celerity approximated a constant. We note that longer separation distances between two locations would yield more accurate wave celerity estimates due to the longer traveling time.

\section{Comparison with theoretical model}

As mentioned in the Introduction, the key objective of this study was to provide the necessary experimental data to verify the existing theory. Here, we shall compare the above experimental results with the model predictions developed in Wang and Shen [33]. However, we shall note again that this theory was developed based on an infinite cover without edge effects, whereas in the present experimental study the floating cover was of a finite extent. The potential influence of the edge effects as mentioned earlier was present in the data. 
6.1 Viscoelastic model by Wang and Shen (2010)

By solving the continuity and momentum equations for a floating viscoelastic continuum over an inviscid water body and matching the stress and velocity conditions at the interfaces, Wang and Shen obtained the dispersion relationship between the angular frequency $\sigma$ and the complex wave number $k=k_{s}+i k_{i}$ in the following manner:

$\left(\sigma^{2}-Q_{c} g k \tanh k H\right) J=0$

with

$Q_{c}=1+\frac{\rho_{1}}{\rho_{2}} \frac{g^{2} k^{2} S_{k} S_{\alpha}-\left(N^{4}+16 k^{6} \alpha^{2} v_{e}^{4}\right) S_{k} S_{\alpha}-8 k^{3} \alpha v_{e}^{2} N^{2}\left(C_{k} C_{\alpha}-1\right)}{g k\left(4 k^{3} \alpha v_{e}^{2} S_{k} C_{\alpha}+N^{2} C_{k} S_{\alpha}-g k S_{k} S_{\alpha}\right)}$

and

$J=g k\left(4 k^{3} \alpha v_{e}^{2} S_{k} C_{\alpha}+N^{2} C_{k} S_{\alpha}-g k S_{k} S_{\alpha}\right)$

where $v_{e}=v+i G / \rho_{1} \sigma$ is the complex viscosity, $\alpha=k^{2}-i \sigma / v_{e}, S_{k}=\sinh (k h), S_{\alpha}=$ $\sinh (\alpha h), C_{k}=\cosh (k h), C_{\alpha}=\cosh (\alpha h)$ and $N=\sigma+2 i k^{2} v_{e}$. The rheological parameters in the complex viscosity $v_{e}$ correspond to the viscoelastic moduli through $G=G^{\prime}$, and $v=G^{\prime \prime} / \sigma \rho_{1}$. In the present experiments with the PDMS covers, $\rho_{1}=940 \mathrm{~kg} / \mathrm{m}^{3}$ was the density of the viscoelastic cover and $\rho_{2}=1000 \mathrm{~kg} / \mathrm{m}^{3}$ was the density of water. The transcendental dispersion relationship was solved numerically using the Muller method [33] with the values of shear modulus and viscosity of the PDMS material used in the experiments. This dispersion relationship has multiple modes, which is common in wave propagation through layered materials, e.g. pure viscous fluid over inviscid water [13]. We chose the mode with its real part closest to the open water case and compared to the wave celerity measured in the experiments. As discussed in Wang and Shen [33] this mode is the dominant one. 
The value of $G^{\prime}$ and $G^{\prime \prime}$ obtained from the frequency sweep analysis is independent of strain applied within the linear viscoelastic regime. Hence the wave frequency was matched with the angular frequency of oscillatory test to get the required $G^{\prime}$ and $G^{\prime \prime}$ from the obtained data. Table 3 summarizes the viscoelastic parameters of the PDMS covers for the range of wave periods in the experiments.

\subsection{Comparison of experimental data and model predictions for viscoelastic cover}

A comparison of the normalized wave number between the experimental data and model predictions by Wang and Shen [33] for the PDMS covers is summarized in Table 4, in which $k_{s i}$, $i=1,2,3$ denotes the real part of the three most significant roots with the least attenuation among the infinite roots obtained as described in [33]. By choosing the one closest to the open water wave number from the three roots, we compared the theoretical results of $\kappa=k_{s} / k_{o}$ with experimental data in Figure 8, where $k_{s}$ is real part of the wave number under the cover and $k_{o}$ is that for open water. Note that $\kappa=1$ for open water, and for wave lengthening $\kappa<1$ and wave shortening $\kappa>1$. In general, within the present range of tested PDMS, the model predicted mostly $\kappa>1$ along the cover which was in agreement with the experimental data. The two cases with $\kappa<1$ were again consistent with the experimental measurements.

Individual plots in Figure 8 correspond to PDMS covers with different rheological properties. Figure $8 \mathrm{a}$ shows the cover with $m_{C A}=4 \%$ thus the lowest shear modulus and viscosity. The model closely predicted the normalized wave number with the shortest wave period of $T=0.5 \mathrm{~s}$. However, noticeable differences can be observed with $\mathrm{T}=0.7 \mathrm{~s}$ and beyond, and in general the model predicted a lower $\kappa$ compared with the experimental measurements. Similar trends can also be identified in the other wave period cases of Figure $8 \mathrm{~b}$ to $8 \mathrm{~d}$. The differences are less for higher shear modulus. Excellent agreement between the model predictions and experiment data can in fact be observed for the two covers with $m_{C A}=8 \%$ and $10 \%$ for $\mathrm{T}=0.6$ to $0.8 \mathrm{~s}$. The differences for the longer wave periods of $0.9 \mathrm{~s}$ and $1.0 \mathrm{~s}$ remained however generally unchanged throughout the tested range. 
Two important reasons might have contributed towards the differences between the theoretical results and experimental data. The first reason was due to the overwash at the leading and trailing edges of the cover, which occurred for the experiments with the shorter wave periods and for covers with larger value of shear modulus, $m_{C A}=10 \%$ (Figure $8 \mathrm{~d}$ and Figure 9). The study by Skene et al [25] showed that the overwash increases with wave steepness. In the present case, the importance of overwash was only noted after the experiments with viscoelastic covers were completed, and we were not able to repeat the experiments with additional edge protection to eliminate the overwash. Since overwash was not observed for most cases with PDMS, the discrepancies were most likely due to a second reason, which was the finite size of the cover. The finite extent was not included in the theoretical model as discussed earlier. To study the wave propagation through a finite floe would require, the inclusion of damped travelling modes from the two free edges and possible pitching motion of the floating cover. From the spectral analysis shown in Section 5.2, the insignificant variation of spectral energy suggested that the edge effects and pitching motion were minimum. Visual observation also confirmed the absence of pitching. Another source of the discrepancies could involve the deviation of the PDMS covers from that of a Voigt model and nonlinear effects for the higher frequency cases.

6.3 Comparison of experimental data and model predictions for the polypropylene cover

As discussed before, the $1.0 \mathrm{~m}$ PP cover was subjected to relatively strong edge effects and pitching. We thus focused instead on the measurements with the $2.45 \mathrm{~m}$ PP cover only. For this case, we tested wave periods from 0.5 to $1.0 \mathrm{~s}$ with increments of $0.05 \mathrm{~s}$. Figure 10 summarizes the comparison between theoretical and experimental results of the dimensionless wave number, in which we also plotted results from the thin-elastic plate theory from Fox and Squire [7] for comparison. The predictions for the two theories were very close, as expected from the asymptotic behavior examined in [33]. Ignoring the viscosity, when the elastic modulus increased the dispersion from Wang and Shen [33] would converge to that of the thin-elastic plate in 7]. As shown in Figure 10, $\kappa$ was always less than 1 which implied wave lengthening. A close agreement was observed between the measurements and theoretical predictions for the 
shorter wave periods from 0.5 to $0.6 \mathrm{~s}$. In this range, with the length of the cover being much longer than the wavelength, we observed visually that pitching was small. Nevertheless, for periods greater than $0.6 \mathrm{~s}$, differences between the measurements and predictions began to be noticeable. This trend was consistent with the observations along the cover discussed earlier in Figure 8. The relative discrepancies between theoretical results and experimental data were greater for the PP case than the PDMS case. With a shear modulus more than three orders of magnitude greater than that of PDMS, the finite size effects of the PP cover, even with a longer cover size, were likely be much greater than the PDMS cover. For the PP cover, to avoid overwash, the installed Styrofoam barrier also changed the boundary condition. In addition, the rheological property of the PP cover did not strictly follow the ideal Voigt model either. These were all potential sources for the observed differences between the theoretical and experiment results.

\section{Conclusions}

In this study, we developed an experimental approach using Polydimethylsiloxane (PDMS) material mixed with oil to produce a viscoelastic float material. The rheological characteristics of this material approximated the Voigt model, thus could be used to verify the viscoelastic model for sea ice by Wang and Shen (2010) [33] which model ice cover as a Voigt material. A range of targeted viscoelastic properties were created by using different amount of curing agent. Due to the large coverage required for the wave flume experiments, special curing procedures were also established for the PDMS preparation beyond the prevalent procedures for small PDMS applications in the literature. Polypropylene (PP) cover with more than three orders of magnitude higher shear modulus than the PDMS covers was also used in the experiments, to contrast the very flexible PDMS covers.

Wave flume experiments showed that wave shortening was observed under the PDMS cover with lower shear modulus for the range of wave periods tested. When the shear modulus was increased, wave lengthening began to be observed for lower wave periods. The model 
predictions by Wang and Shen [33] were generally consistent with the experimental data, both in terms of the switching of normalized wavelength from less than to greater than 1 , as well as the magnitude of the change. Wave lengthening was observed for the PP cover for all cases studied, and approached the open water wave celerity with increase in the wave period. These results were also consistent with theoretical predictions.

For both PDMS and PP covers, comparisons between theoretical and experimental results were better for shorter wave periods, despite the higher wave steepness and thus some nonlinear effects. Discrepancies for longer wave periods can be attributed to the finite extent of the cover in the experiments and edge barriers for PP cover. The rheometer measurements for PDMS and PP cover showed that both of them were not strictly linear Voight material. The deviations of experimental results from the theoretical model might be due to this discrepancy which was more for the PP cover than PDMS.

Overall, the present study provides a general confirmation of the viscoelastic theory by Wang and Shen [33] in terms of the dispersion relationship and also illustrates the level of predictive accuracy of the theory within a range of viscoelastic properties and wave characteristics. The next step would be to examine theoretically the influence due to finite extent of the cover, and also experimentally determine the wave attenuation under the viscoelastic cover. Despite these efforts, there is still a major gap between the current viscoelastic theory, even verified experimentally, and the ice cover in the MIZ. To determine whether real ice covers that consist a variety of different ice features can or cannot be modeled as a Voigt or other types of viscoelastic continuum, different approaches may be taken: either from using first principles to derive such a theory, or from a large amount of direct measurements in the laboratory or from field experiments. For both cases, considerable future work needs to be done to fill this gap.

\section{Acknowledgements}


The authors gratefully acknowledge the funding provided by Ministry of Education, Singapore through the AcRF Tier 2 Grant No. MOE2013-T2-1-054. This work is supported in part by the US Office of Naval Research Grant \#N00014-13-1-0294 and US Office of Naval Research Global Grant No: N62909-15-1-2069. The authors would like to thank Sukun Cheng at Clarkson University for providing the code for the viscoelastic model [33] and Malavarayan Sankarasubramanian at Clarkson University for measuring the polypropylene parameters. 


\section{REFERENCES}

[1] Andrianov, A. (2005). Hydroelastic analysis of very large floating structures. PhD Thesis, Delft University of Technology.

[2] Bennetts, L. G., Alberello, A., Meylan, M. H., Cavaliere, C., Babanin, A. V., and Toffoli, A. (2015). An idealised experimental model of ocean surface wave transmission by an ice floe. Ocean Modelling, 96, 85-92.

[3] Bennetts, L. G., and Williams, T. D. (2015). Water wave transmission by an array of floating discs. In Proceedings of the Royal Society of London A: Mathematical, Physical and Engineering Sciences. Vol. 471, No. 2173, p. 20140698.

[4] Bennetts, L. G., and Squire, V. A. (2012). On the calculation of an attenuation coefficient for transects of ice-covered ocean. In Proc. R. Soc. A, Vol. 468, No. 2137, pp. 136-162.

[5] Chen, X. J., Wu, Y. S., Cui, W. C., and Jensen, J. J. (2006). Review of hydroelasticity theories for global response of marine structures. Ocean Engineering, 33(3), 439-457.

[6] Deng, Z., Sree, D., Prabowo, F., Law, A. W. K., and Shen, H. H. (2015). An Experimental Study on the Attenuation of Surface Waves due to a Viscoelastic Floating Cover. Proceeding, 7th International Conference on Fluid Mechanics, Qingdao, China.

[7] Fox, C., and Squire, V. A. (1990). Reflection and transmission characteristics at the edge of shore fast sea ice. Journal of Geophysical Research: Oceans, 95(C7), 11629-11639.

[8] Fujii, T. (2002). PDMS-based microfluidic devices for biomedical applications. Microelectronic Engineering, 61, 907-914.

[9] Greenhill, A. G. (1886). Wave motion in hydrodynamics. American Journal of Mathematics, 62-96.

[10] Hermans, A. J. (2003). Interaction of free-surface waves with a floating dock.Journal of engineering mathematics, 45(1), 39-53.

[11] Hermans, A. J. (2004). Interaction of free-surface waves with floating flexible strips. Journal of engineering mathematics, 49(2), 133-147.

[12] Hermans, A. J. (2011). Flexible Floating Platform. In Water Waves and Ship Hydrodynamics (pp. 87-102).

[13] Keller, J. B. (1998). Gravity waves on ice-covered water. Journal of Geophysical Research, 103(C4), 7663-7669. 
[14] Kohout, A. L., and Meylan, M. H. (2008). An elastic plate model for wave attenuation and ice floe breaking in the marginal ice zone. Journal of Geophysical Research: Oceans, 113(C9).

[15] Meylan, M. H., and V. A. Squire (1994), The response of ice floes to ocean waves, J. Geophys. Res., 99(C1): 899-900.

[16] Meylan, M. H., and V. A. Squire (1996), Response of a circular ice floe to ocean waves, J. Geophys. Res., 101(C4): 8869-8884.

[17] Meylan, M.H., Squire, V.A., and Fox, C. (1997) Toward realism in modeling ocean wave behavior in marginal ice zones, J. Geophys. Res., 102(C10):22981-22991.

[18] Montiel, F., Bonnefoy, F., Ferrant, P., Bennetts, L. G., Squire, V. A., and Marsault, P. (2013). Hydroelastic response of floating elastic discs to regular waves. Part 1. Wave basin experiments. Journal of Fluid Mechanics 723: 604-628.

[19] Montiel, F., Bennetts, L. G., Squire, V. A., Bonnefoy, F., and Ferrant, P. (2013). Hydroelastic response of floating elastic discs to regular waves. Part 2. Modal analysis. Journal of Fluid Mechanics, 723, 629-652.

[20] Montiel, F., Squire, V. A., and Bennetts, L. G. (2016). Attenuation and directional spreading of ocean wave spectra in the marginal ice zone. Journal of Fluid Mechanics, 790, 492-522.

[21] Mosig, J. E. M., Montiel, F. and Squire, V.A. (2015) Comparison of viscoelastic-type models for ocean wave attenuation in ice-covered seas, J. Geophys. Res. Oceans, 120:6072-6090.

[22] Prabowo, F., Sree, D., Law, A. W. K., and Shen, H. H. (2014). A Laboratory Study of Wave-Ice Interactions in the Marginal Ice Zone using Polydimethylsiloxane (PDMS) as a Viscoelastic Model. 22nd IAHR International Symposium on Ice.

[23] Schramm, G. (1994). A practical approach to rheology and rheometry. Karlsruhe: Haake.

[24] Sendil, U., and Graf, W. H. (1974). Transmission of regular waves past floating plates. Coastal Engineering Proceedings, 1(14).

[25] Skene, D. M., Bennetts, L. G., Meylan, M. H., and Toffoli, A. (2015). Modelling water wave overwash of a thin floating plate. Journal of Fluid Mechanics, 777, R3. 
[26] Smith, L. C., and Stephenson, S. R. (2013). New Trans-Arctic shipping routes navigable by midcentury. Proc Natl Acad Sci U S A, 110(13), E1191-1195.

[27] Squire, V. A. (2007). Of ocean waves and sea-ice revisited. Cold Regions Science and Technology, 49(2), 110-133.

[28] Squire, V. A., and Allan, A. J., 1980. Propagation of flexural gravity waves in sea ice. Proceedings of the Arctic Ice Dynamics Joint Experiment International Commission on Snow and Ice Symposium (pp. 327-338) (R. S. Pritchard, Ed.). Seattle, Wash: Univ. of Wash. Press.

[29] Stroeve, J. C., Kattsov, V., Barrett, A., Serreze, M., Pavlova, T., Holland, M., and Meier, W. N. (2012). Trends in Arctic sea ice extent from CMIP5, CMIP3 and observations. Geophysical Research Letters, 39(16).

[30] Tolman, H., Accensi, M., Alves, H., Ardhuin, F., Bidlot, J., Booij, N., and Filipot, J. F. (2014). User manual and system documentation of Wavewatch III® version 4.18. Tech. Note 316, NOAA/NWS/NCEP/MMAB.

[31] Wadhams, P. The effect of a sea ice cover an ocean surface waves. PhD thesis, University of Cambridge, 1973.

[32] Wadhams, P. (1986). The seasonal ice zone. The geophysics of sea ice, Springer US, 825-991.

[33] Wang, R., and Shen, H. H. (2010). Gravity waves propagating into an ice-covered ocean: A viscoelastic model. Journal of Geophysical Research, 115(C6).

[34] Wang, C.M., Watanabe, E., and Utsunomiya, T. (2008) (Editors) Very large floating structures. Taylor and Francis, London and New York. ISBN 0-203-93460-1.

[35] Watanabe, E., Wang, C.M., Utsunomiya, T., and Moan, T. (2004a) Very large floating structures: applications, analysis and design. Centre for Offshore Research and Engineering, National University of Singapore, CORE Report No. 2004-02.

[36] Watanabe, E., Utsunomiya, T., and Wang, C.M., (2004b) Hydroelastic analysis of pontoon-type VLFS: a literature survey. Eng. Struct. 26 (2), 245-256.

[37] Weitz, M., and Keller, J.B. (1950) Reflection of water waves from floating ice in water of finite depth, Communs. Pre Appl. Math., 3, 305-318. 
[38] Williams, T.D., Bennetts, L.G., Squire, V.A., Dumont, D., and Bertino, L. (2013a) Wave-ice interactions in the marginal ice zone. Part 1: Theoretical foundations. Ocean Modelling, 71:81-91.

[39] Williams, T.D., Bennetts, L.G., Squire, V.A., Dumont, D., and Bertino, L. (2013b) Wave-ice interactions in the marginal ice zone. Part 2: Numerical implementation and sensitivity studies along 1D transects of the ocean surface. Ocean Modelling, 71:92-101. 


\section{LIST OF SYMBOLS}

c

Wave celerity in open water

$c_{s} \quad$ Wave celerity along the cover

d

Water depth

G

Shear modulus

$G^{\prime}$

Storage modulus

$G^{\prime \prime}$

Loss modulus

$h$

Thickness of viscoelastic cover

$a$

Wave amplitude

$k$

Complex wave number along the floating cover, $k=k_{s}+i k_{i}$

$k_{S}$

Real part of complex wave number

$k_{i}$

Imaginary part of complex wave number

$k_{o}$

Open water wave number

$m_{o}$

Mass percentage of white oil

$m_{C A}$

Mass percentage of curing agent

$M_{O}$

Mass of white oil

$M_{C A}$

Mass of curing agent

$M_{B}$

Mass of base (prepolymer)

t

Time

$\mathrm{T}$

Wave period

Distance measured along the cover from the leading edge

Mean of the 3 repetitions 


$\begin{array}{ll}E & \text { Standard deviation } \\ \eta & \text { Vertical displacement of cover } \\ v & \text { Kinematic viscosity } \\ \rho & \text { Density of material tested } \\ \rho_{1} & \text { Density of viscoelastic cover } \\ \rho_{2} & \text { Density of water } \\ \gamma & \text { Deformation/shear strain } \\ \tau & \text { Stress } \\ \sigma & \text { Wave angular frequency } \\ \kappa & \text { Normalized wave number }=k_{s} / k_{o} \\ \xi & \text { Relaxation time } \\ \Delta x_{i} & \text { Distance of the } i^{\text {th }} \text { ultrasound sensor from US1 } \\ \Delta t & \text { Time lapse }\end{array}$




\section{$\underline{\text { LIST OF TABLES }}$}

Table 1: Ratio of loss modulus to storage modulus for different $m_{C A}$.

Table 2: Input wave characteristics.

Table 3: Variation of the viscoelastic properties of PDMS material for different $m_{C A}$.

Table 4: Comparison of wave numbers obtained from wave experiment and theoretical model (Wang and Shen, 2010).

\section{LIST OF FIGURES}

Figure 1: Viscoelastic properties against angular frequency. Curing Period: circles = 1 week; asterisk $=2$ week; diamond $=3$ week. $m_{C A}=$ (a) $4 \%$, (b) $6 \%$, (c) $8 \%$ and, (d) $10 \%$.

Figure 2: Schematic diagram of the wave flume.

Figure 3: Time series of the wave record at US1 $(\mathrm{T}=0.7 \mathrm{~s})$ for three tests with identical wave generator setting. The boxed portion was used for analysis. Repeatability of tests is checked since the three time series overlapped exactly.

Figure 4: Wave celerity under the PDMS cover. The solid line represents the open water case. T $=$ (a) $1.0 \mathrm{~s}$, (b) $0.7 \mathrm{~s}$, and (c) $0.5 \mathrm{~s}$.

Figure 5: Spectral analysis of the surface elevation at different locations along the cover $(\mathrm{T}=0.7$ s): (a) PDMS $\left(m_{C A}=10 \%\right)$ and (b) $1.0 \mathrm{~m}$ PP cover. The locations are marked with A-E from the leading edge, with distance in meters. The data were vertically displaced by increments of 0.5 and 0.25 for (a) and (b) respectively to aid visualization.

Figure 6: Spectral analysis of the surface elevation at different locations for $2.45 \mathrm{~m}$ long PP cover. $\mathrm{T}=$ (a) $0.7 \mathrm{~s}$ and, (b) $\mathrm{T}=0.5 \mathrm{~s}$. The data were vertically displaced from $\mathrm{A}$ to $\mathrm{G}$ by increments of 0.1 to aid visualization.

Figure 7: Wave celerity along the length of the long PP cover. Solid line = open water wave celerity; dashed line $=$ model predictions (Wang and Shen, 2010) and circles = experimental data. $\mathrm{T}=$ (a) $0.5 \mathrm{~s}$, (b) $0.6 \mathrm{~s}$, (c) $0.7 \mathrm{~s}$, (d) $0.8 \mathrm{~s}$, (e) $0.9 \mathrm{~s}$ and, (f) $1.0 \mathrm{~s}$. 
Figure 8: Normalized wave number under the PDMS cover against wave period. Dashed line = open water case, circles $=$ experimental data, and asterisk $=$ model predictions (Wang and Shen, 2010). $m_{C A}=$ (a) $4 \%$, (b) $6 \%$, (c) $8 \%$ and, (d) $10 \%$. The symbol enclosed in a box corresponds to the case when overwash was observed.

Figure 9: Over wash at the leading edge of the PDMS cover for the higher frequency case, $\mathrm{T}=$ $0.5 \mathrm{~s}$ and $m_{C A}=10 \%$.

Figure 10: Normalized wave number under the $2.45 \mathrm{~m}$ long PP cover. Solid line $=$ open water, asterisk $=$ model predictions (Wang and Shen, 2010), and circles = experimental data. 
Table 1: Ratio of loss modulus to storage modulus for different $m_{C A}$.

\begin{tabular}{|c|c|c|c|c|}
\hline \multirow{2}{*}{$\mathrm{T}(\mathrm{s})$} & \multicolumn{4}{|c|}{$G^{\prime \prime} / G^{\prime}$} \\
\cline { 2 - 5 } & $4 \%$ & $6 \%$ & $8 \%$ & $10 \%$ \\
\hline 0.5 & 0.1161 & 0.0544 & 0.0428 & 0.0331 \\
\hline 0.6 & 0.1096 & 0.0515 & 0.0407 & 0.0313 \\
\hline 0.7 & 0.1039 & 0.0489 & 0.0387 & 0.0297 \\
\hline 0.8 & 0.0992 & 0.0467 & 0.0372 & 0.0285 \\
\hline 0.9 & 0.0955 & 0.0450 & 0.0359 & 0.0275 \\
\hline 1.0 & 0.0924 & 0.0436 & 0.0349 & 0.0267 \\
\hline
\end{tabular}


Table 2: Input wave characteristics.

\begin{tabular}{|c|c|c|c|}
\hline $\mathrm{T}(\mathrm{s})$ & $\lambda(\mathrm{m})$ & $k_{o}(\mathrm{rad} / \mathrm{m})$ & $k_{o} a$ \\
\hline 0.5 & 0.390 & 16.08 & 0.137 \\
\hline 0.6 & 0.560 & 11.19 & 0.095 \\
\hline 0.7 & 0.755 & 8.32 & 0.071 \\
\hline 0.8 & 0.960 & 6.53 & 0.056 \\
\hline 0.9 & 1.168 & 5.37 & 0.046 \\
\hline 1.0 & 1.373 & 4.57 & 0.039 \\
\hline
\end{tabular}


Table 3: Variation of the viscoelastic properties of PDMS material for different $m_{C A}$.

\begin{tabular}{|c|c|c|c|c|c|c|c|c|}
\hline \multirow{2}{*}{$\mathrm{T}(\mathrm{s})$} & \multicolumn{4}{|c|}{$G(\mathrm{~Pa})$} & \multicolumn{4}{c|}{$v=\mu / \rho_{1}\left(\mathrm{~m}^{2} / \mathrm{s}\right)$} \\
\cline { 2 - 9 } & $4 \%$ & $6 \%$ & $8 \%$ & $10 \%$ & $4 \%$ & $6 \%$ & $8 \%$ & $10 \%$ \\
\hline 0.5 & 22465 & 55854 & 89542 & 146442 & 0.22 & 0.26 & 0.32 & 0.41 \\
\hline 0.6 & 22248 & 55565 & 89181 & 146081 & 0.25 & 0.29 & 0.37 & 0.46 \\
\hline 0.7 & 220033 & 55333 & 88878 & 145722 & 0.27 & 0.32 & 0.41 & 0.51 \\
\hline 0.8 & 21851 & 55151 & 88635 & 145418 & 0.29 & 0.35 & 0.45 & 0.56 \\
\hline 0.9 & 21709 & 55009 & 88446 & 145182 & 0.32 & 0.38 & 0.48 & 0.61 \\
\hline 1.0 & 21595 & 54893 & 88292 & 144989 & 0.34 & 0.41 & 0.52 & 0.65 \\
\hline
\end{tabular}


Table 4: Comparison of wave numbers obtained from wave experiment and theoretical model (Wang and Shen, 2010).

\begin{tabular}{|c|c|c|c|c|c|c|c|c|}
\hline \multirow{4}{*}{$\mathrm{T}(\mathrm{s})$} & \multicolumn{8}{|c|}{ Real part of wave number ( $\mathrm{rad} / \mathrm{m})$} \\
\hline & \multicolumn{4}{|c|}{$m_{C A}=4 \%$} & \multicolumn{4}{|c|}{$m_{C A}=6 \%$} \\
\hline & Experiment & \multicolumn{3}{|c|}{ Wang and Shen (2010) } & Experiment & \multicolumn{3}{|c|}{ Wang and Shen (2010) } \\
\hline & $k_{s}$ & $k_{s 1}$ & $k_{s 2}$ & $k_{s 3}$ & $k_{S}$ & $k_{s 1}$ & $k_{s 2}$ & $k_{s 3}$ \\
\hline 0.5 & 17.220 & 1.278 & 17.541 & 2.558 & 16.455 & 0.814 & 1.628 & 16.386 \\
\hline 0.6 & 12.617 & 12.301 & 1.071 & 2.143 & 11.959 & 0.680 & 12.008 & 1.361 \\
\hline 0.7 & 9.581 & 8.956 & 0.923 & 1.847 & 9.063 & 8.888 & 0.584 & 1.169 \\
\hline 0.8 & 7.315 & 6.902 & 0.811 & 1.623 & 6.980 & 6.882 & 0.512 & 1.025 \\
\hline 0.9 & 5.871 & 5.595 & 0.724 & 1.448 & 5.884 & 5.587 & 0.456 & 0.912 \\
\hline 1.0 & 4.907 & 4.716 & 0.653 & 1.307 & 4.866 & 0.411 & 4.711 & 0.822 \\
\hline \multirow{3}{*}{$\mathrm{T}(\mathrm{s})$} & \multicolumn{4}{|c|}{$m_{C A}=8 \%$} & \multicolumn{4}{|c|}{$m_{C A}=10 \%$} \\
\hline & Experiment & \multicolumn{3}{|c|}{ Wang and Shen (2010) } & Experiment & \multicolumn{3}{|c|}{ Wang and Shen (2010) } \\
\hline & $k_{s}$ & $k_{s 1}$ & $k_{s 2}$ & $k_{s 3}$ & $k_{s}$ & $k_{s 1}$ & $k_{s 2}$ & $k_{s 3}$ \\
\hline 0.5 & 15.640 & 0.643 & 1.287 & 15.654 & 13.694 & 0.503 & 1.006 & 14.825 \\
\hline 0.6 & 11.653 & 0.537 & 1.075 & 11.771 & 11.308 & 0.420 & 0.840 & 11.448 \\
\hline 0.7 & 8.962 & 0.461 & 8.828 & 0.923 & 8.751 & 0.360 & 8.735 & 0.721 \\
\hline 0.8 & 6.848 & 0.404 & 6.865 & 0.808 & 6.934 & 0.316 & 0.631 & 6.838 \\
\hline 0.9 & 5.789 & 0.360 & 5.582 & 0.719 & 5.789 & 0.281 & 0.561 & 5.573 \\
\hline 1.0 & 4.838 & 0.324 & 4.709 & 0.648 & 4.829 & 0.253 & 0.506 & 4.705 \\
\hline
\end{tabular}



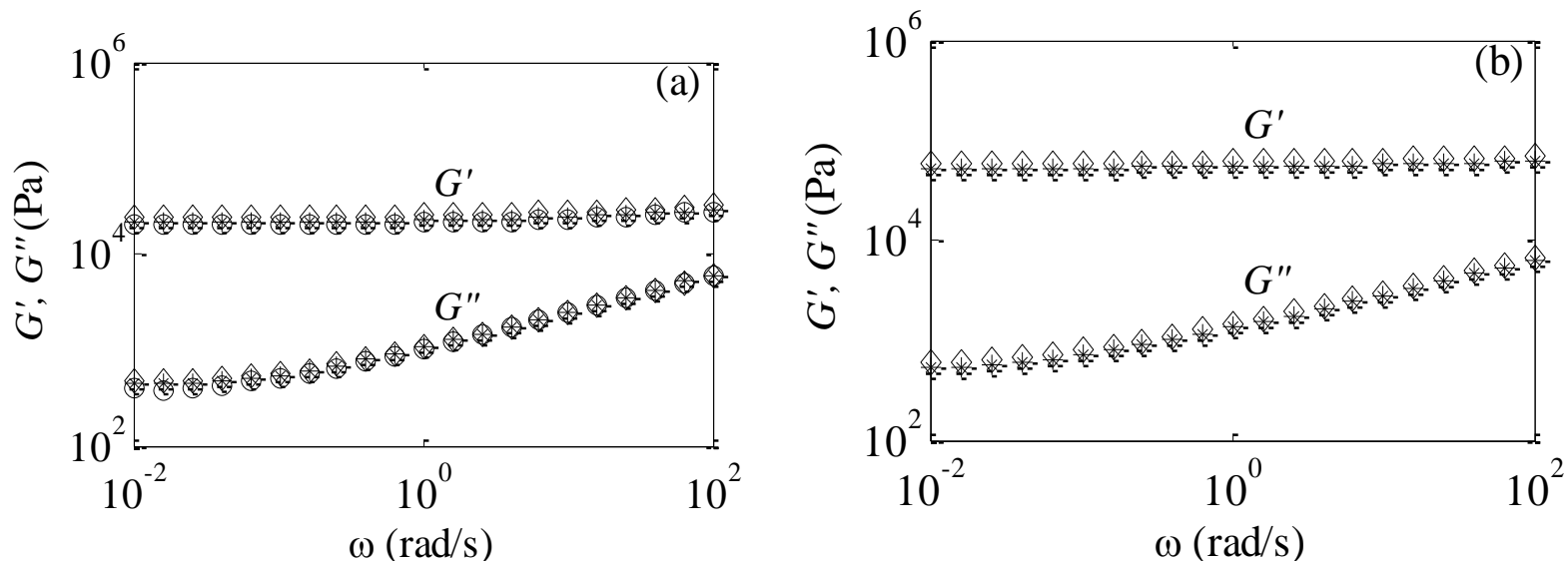

1
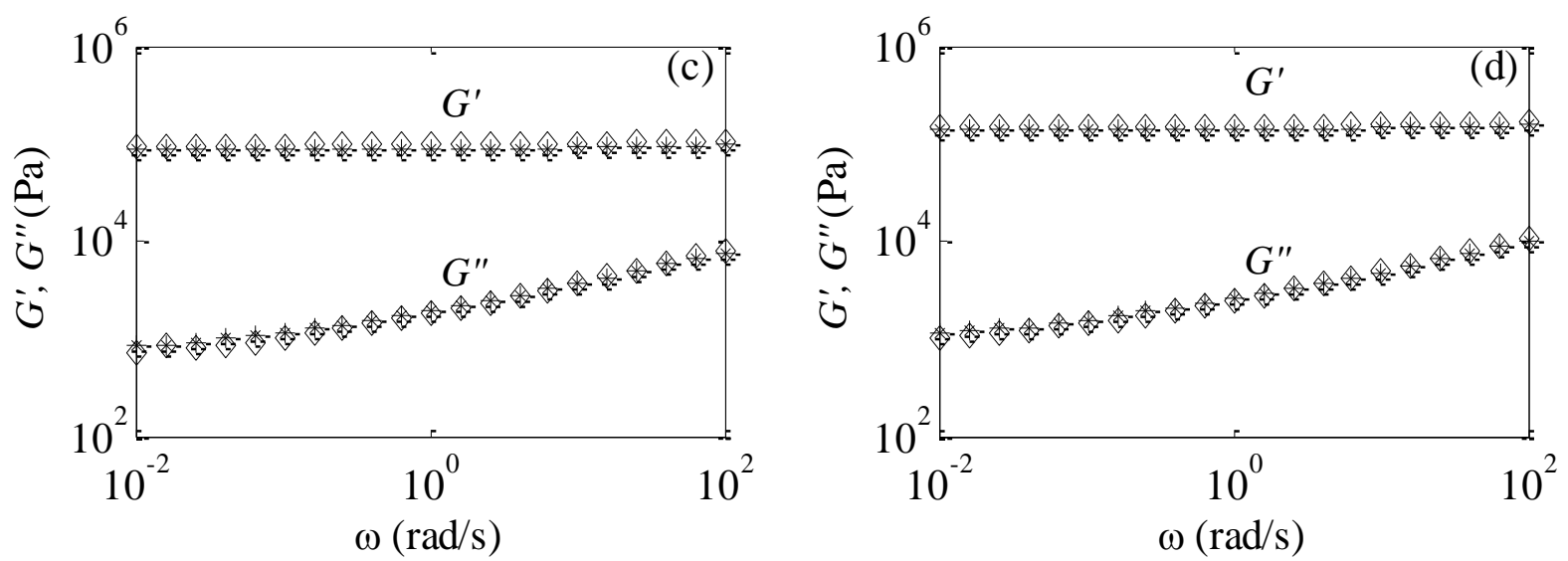

3 Figure 1: Viscoelastic properties against angular frequency. Curing Period: circles = 1 week; asterisk $=2$ week; diamond $=3$ week. $m_{C A}=$ (a) $4 \%$, (b) $6 \%$, (c) $8 \%$ and, (d) $10 \%$. 

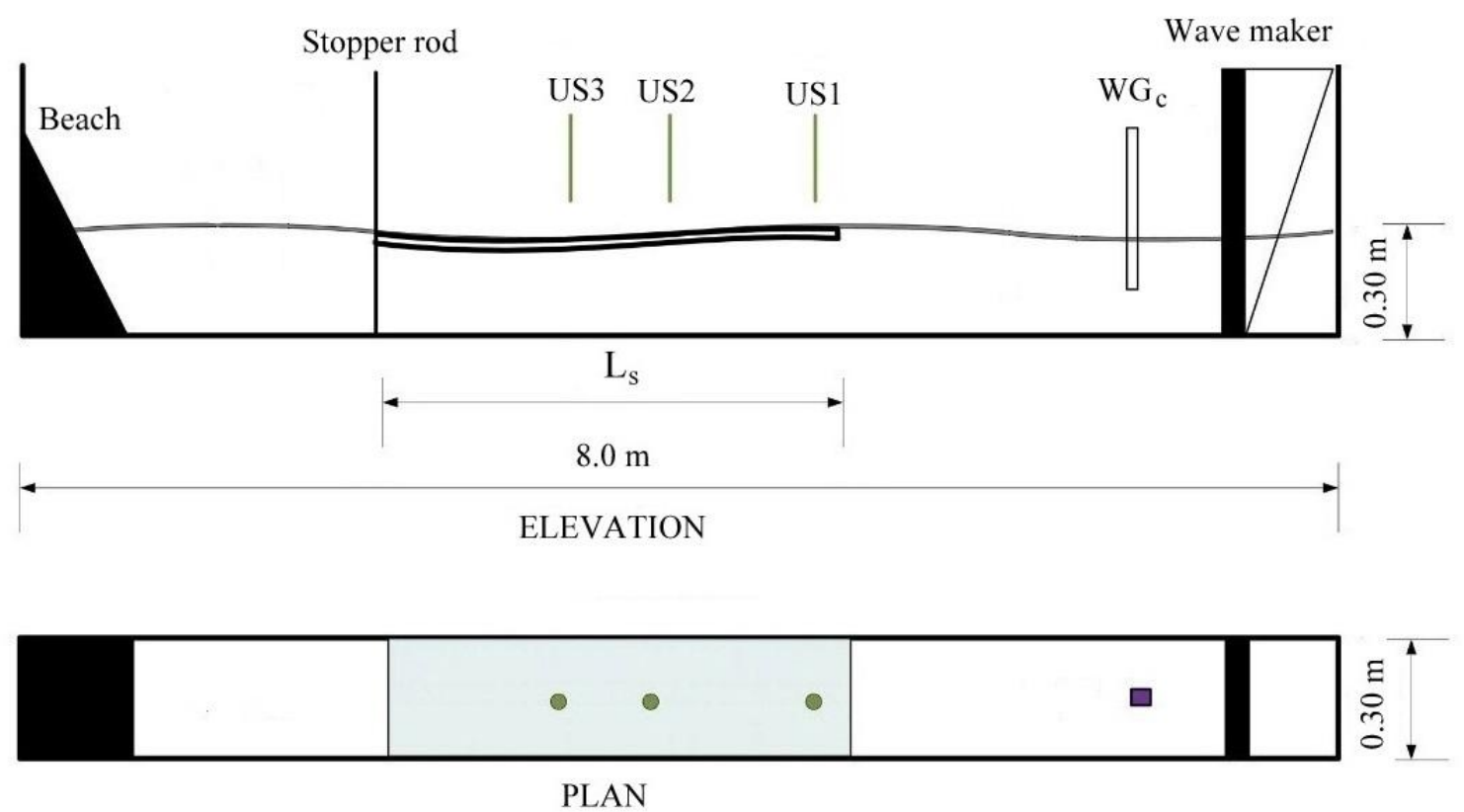

6

Figure 2: Schematic diagram of the wave flume. 


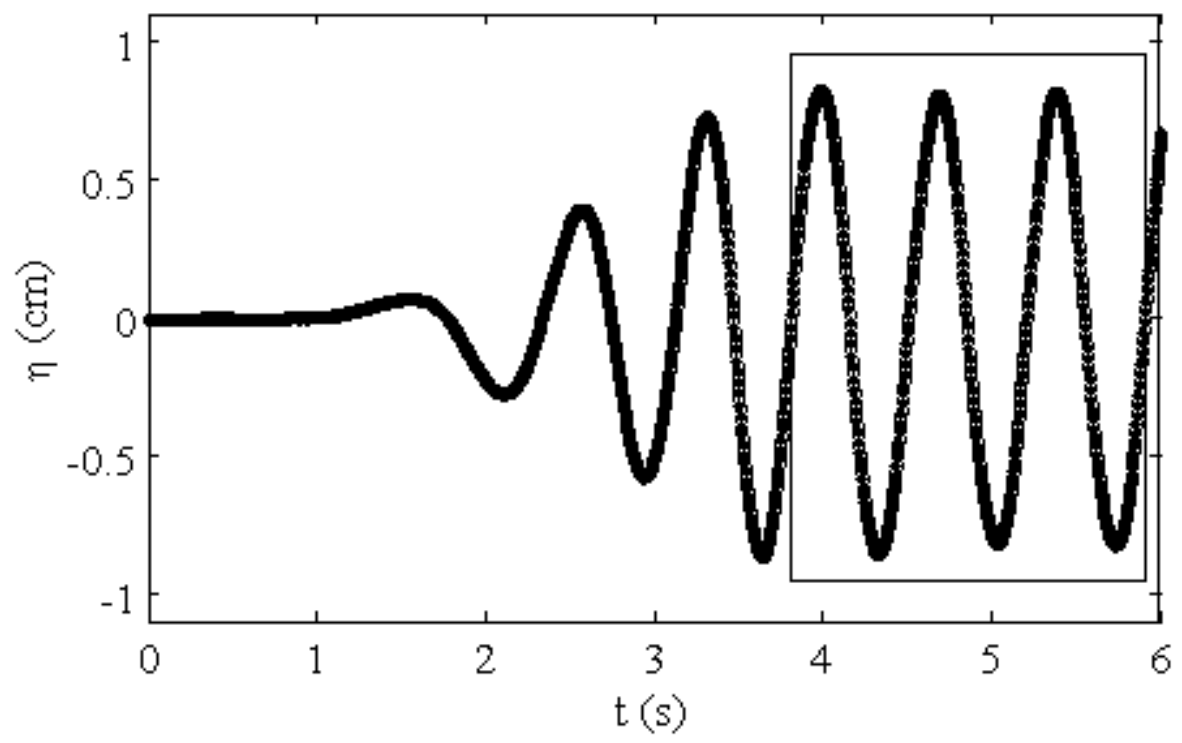

10

Figure 3: Time series of the wave record at US1 $(T=0.7 \mathrm{~s})$ for three tests with identical wave

12 generator setting. The boxed portion was used for analysis. Repeatability of tests is checked since the three-time series overlapped exactly.

14 


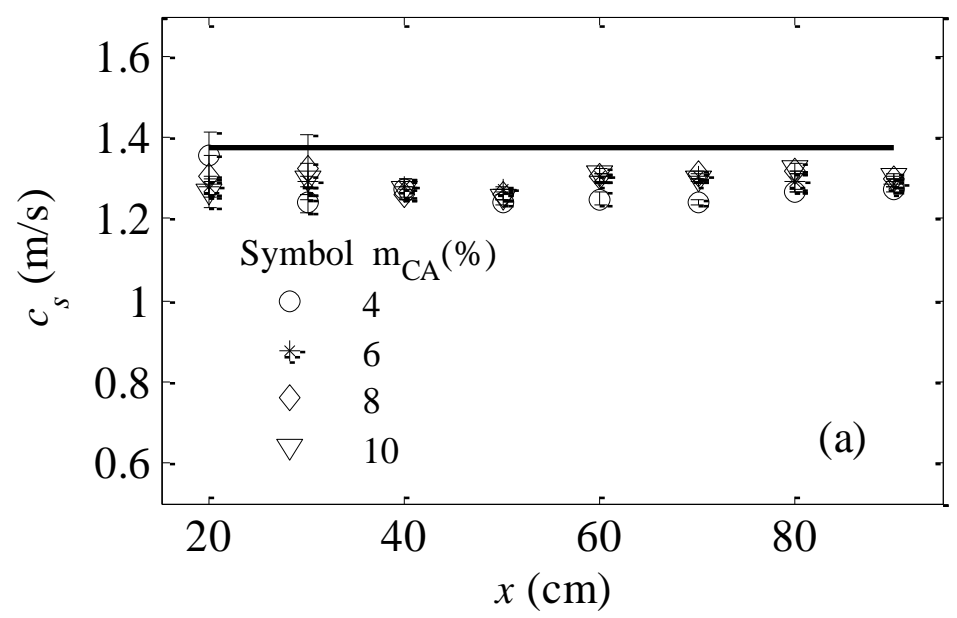

16
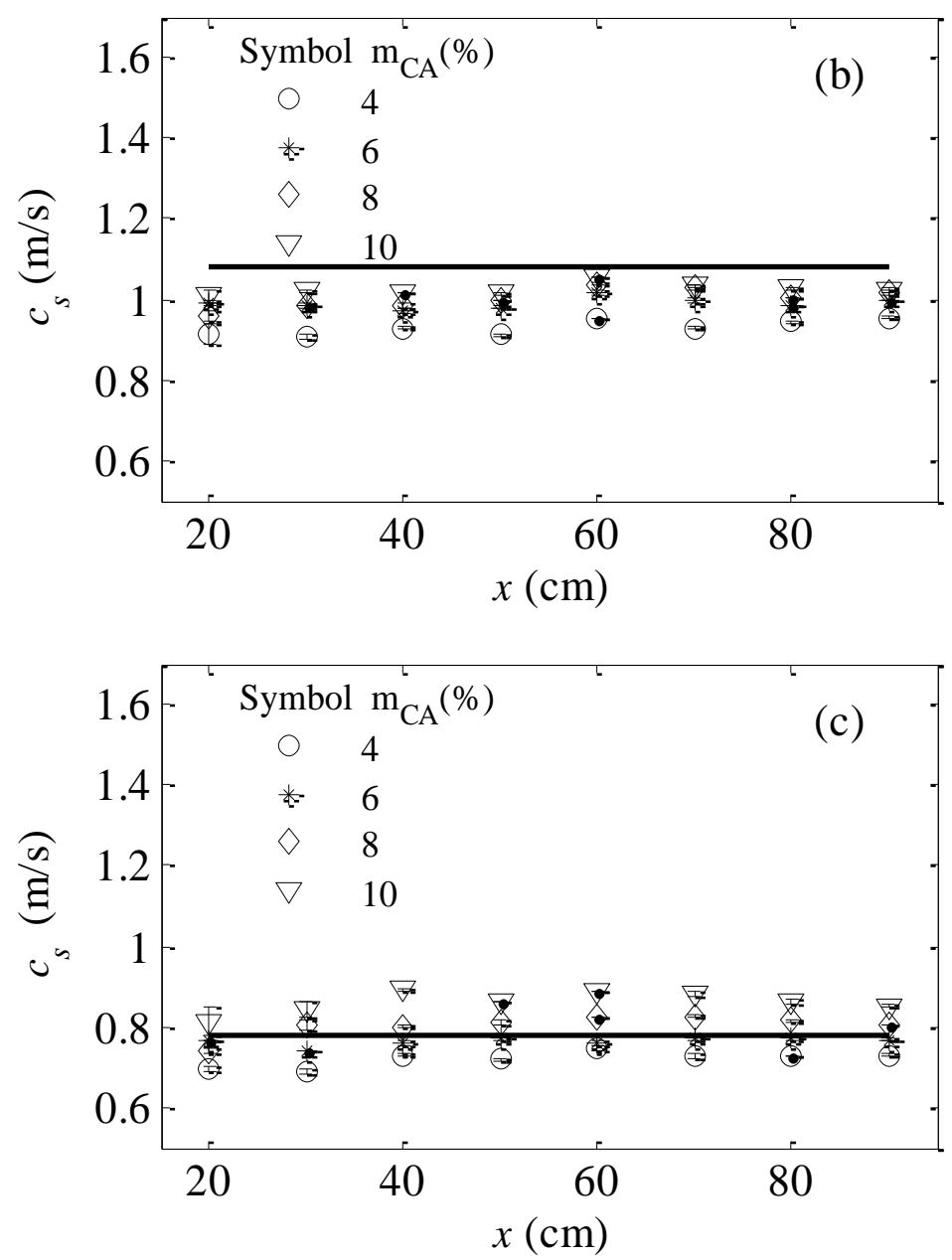

18 Figure 4: Wave celerity under the PDMS cover. The solid line represents the open water case. T 19 $=$ (a) $1.0 \mathrm{~s}$, (b) $0.7 \mathrm{~s}$, and (c) $0.5 \mathrm{~s}$. 

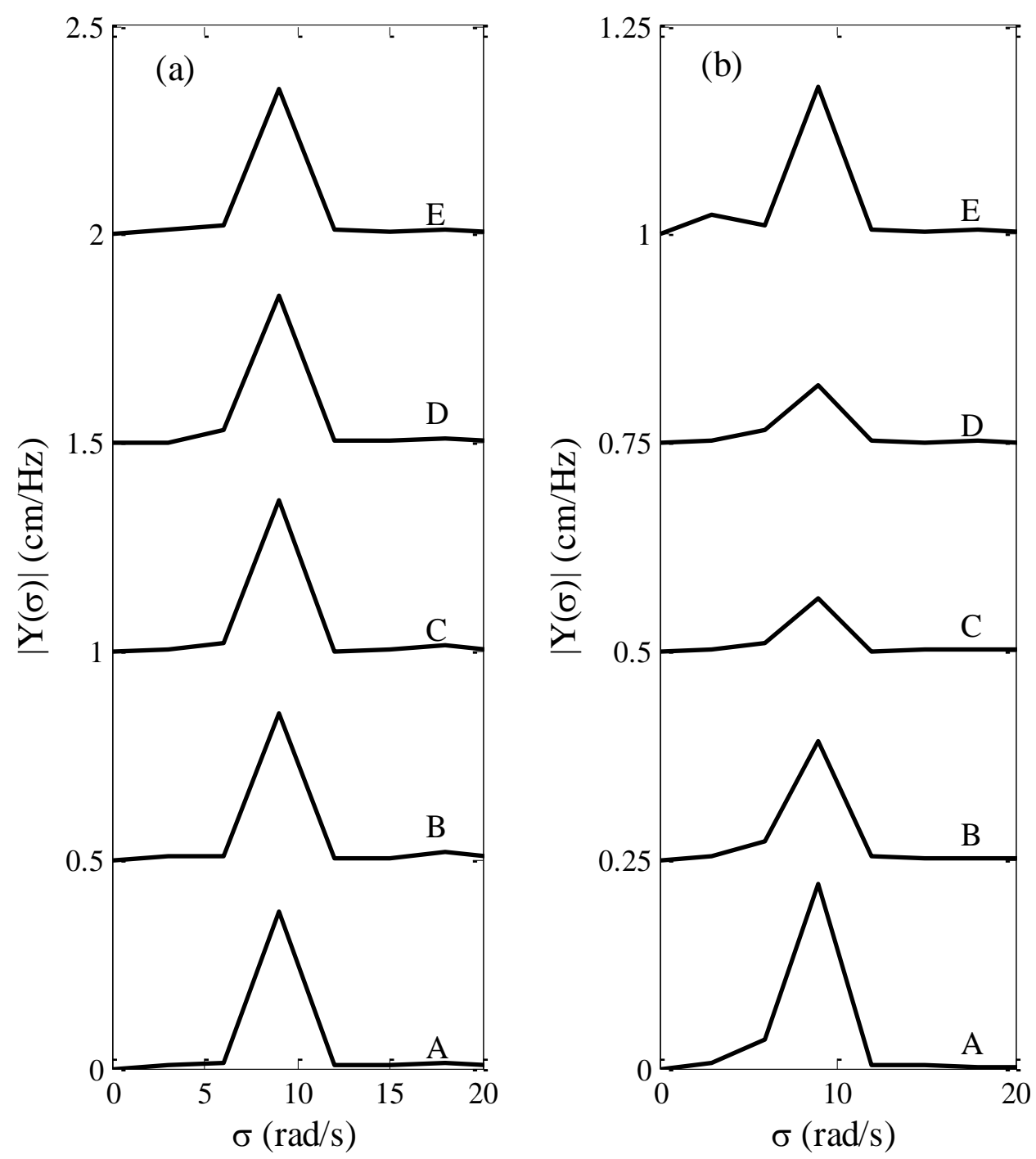

20

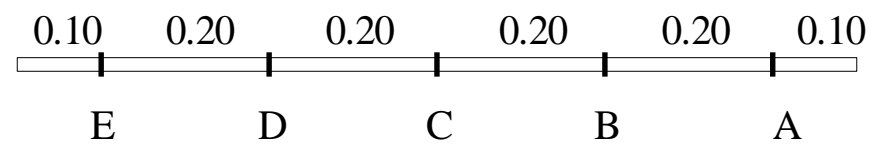

21

22 Figure 5: Spectral analysis of the surface elevation at different locations along the cover $(\mathrm{T}=0.7$

23 s): (a) PDMS $\left(m_{C A}=10 \%\right)$ and (b) $1.0 \mathrm{~m}$ PP cover. The locations are marked with A-E from the 24 leading edge, with distance in meters. The data were vertically displaced by increments of 0.5 25 and 0.25 for (a) and (b) respectively to aid visualization. 

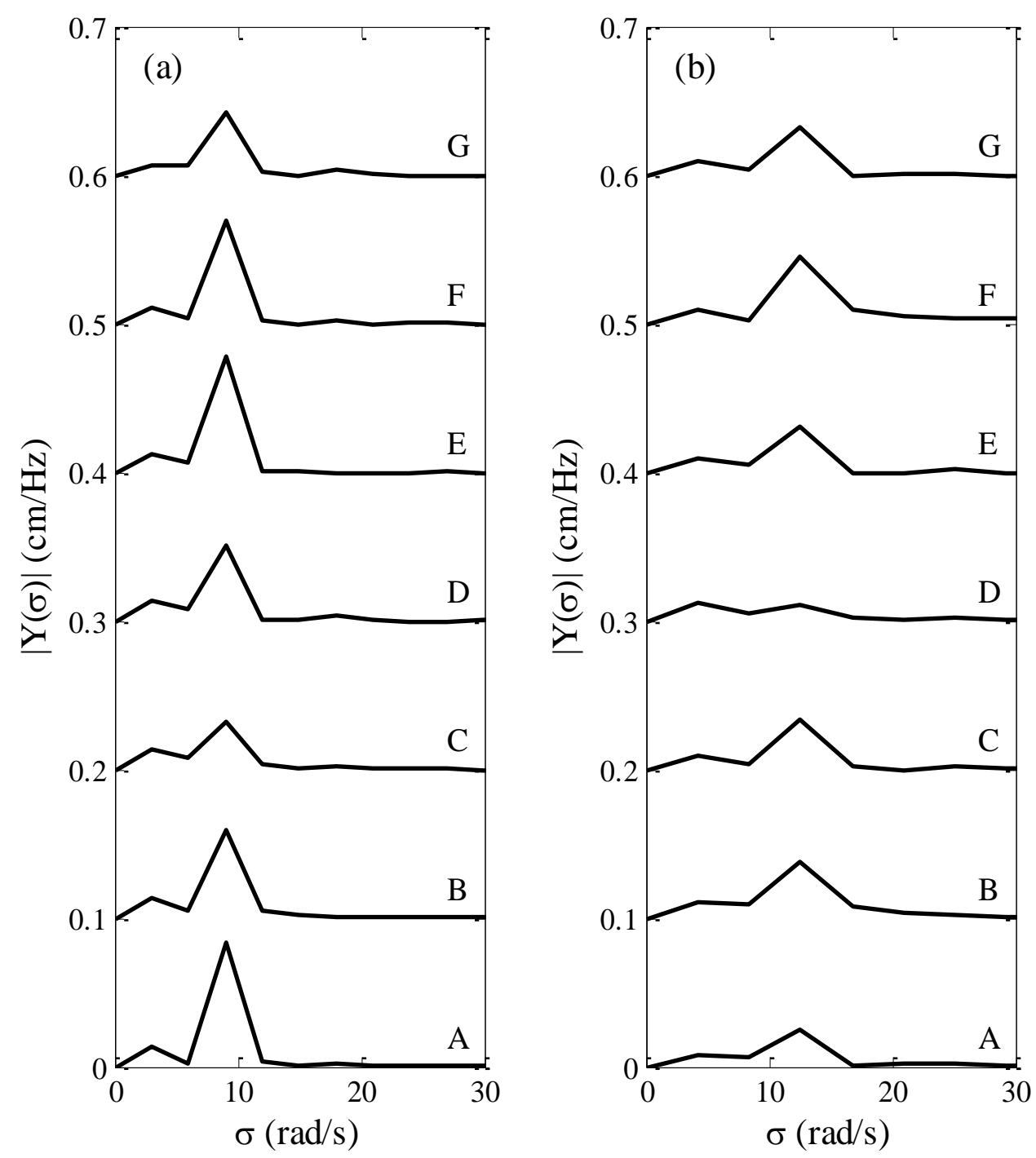

26

27

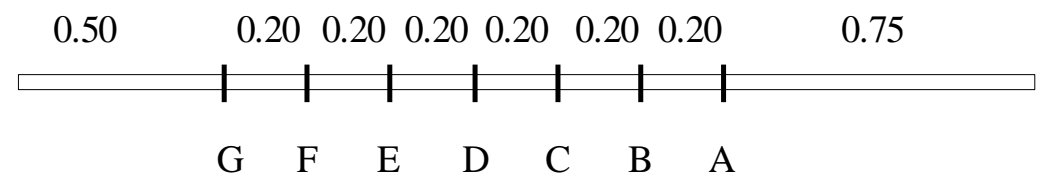

28 Figure 6: Spectral analysis of the surface elevation at different locations for $2.45 \mathrm{~m}$ long PP cover. $\mathrm{T}=$ (a) $0.7 \mathrm{~s}$ and, (b) $\mathrm{T}=0.5 \mathrm{~s}$. The data were vertically displaced from $\mathrm{A}$ to $\mathrm{G}$ by 30 increments of 0.1 to aid visualization. 

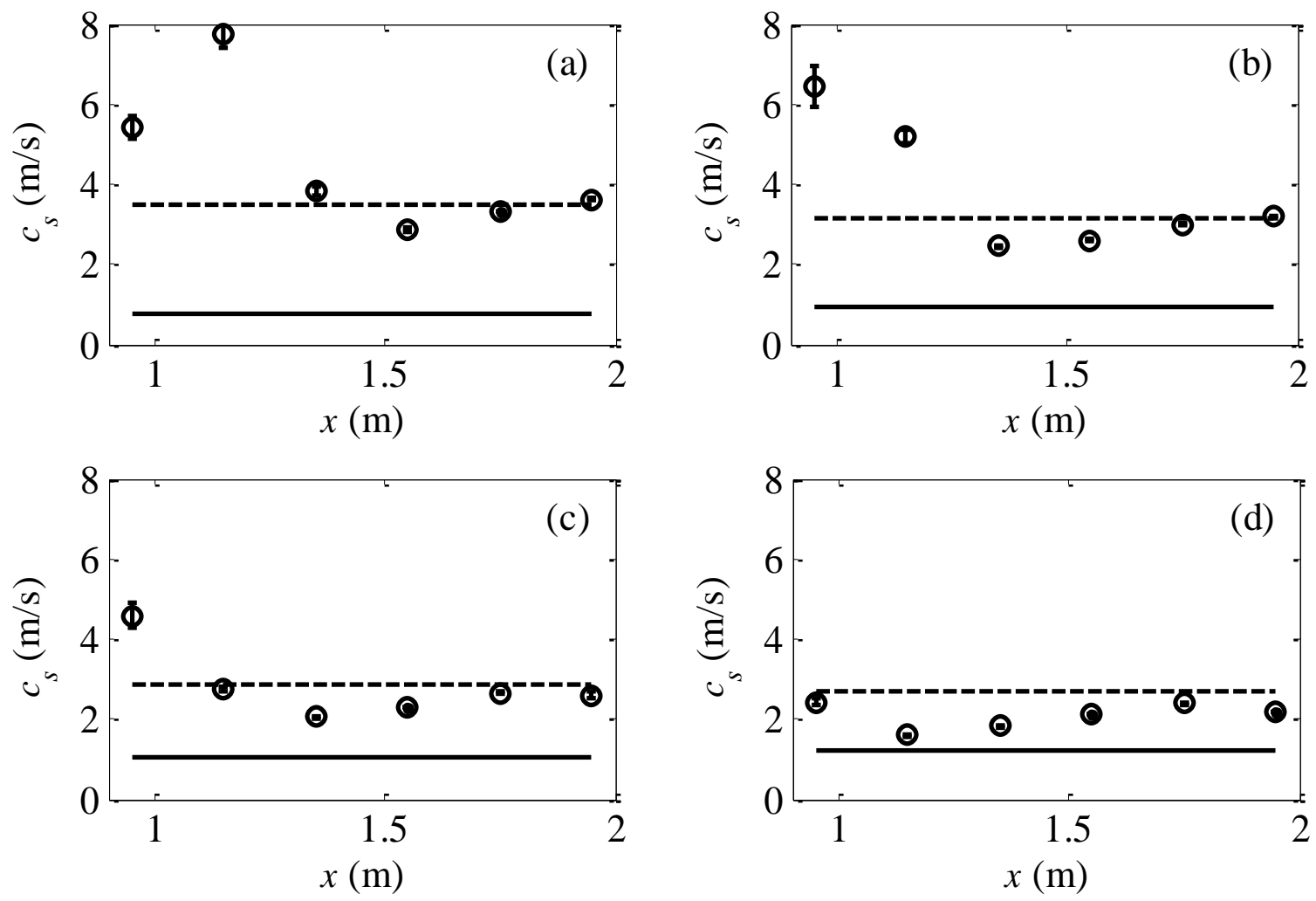

34
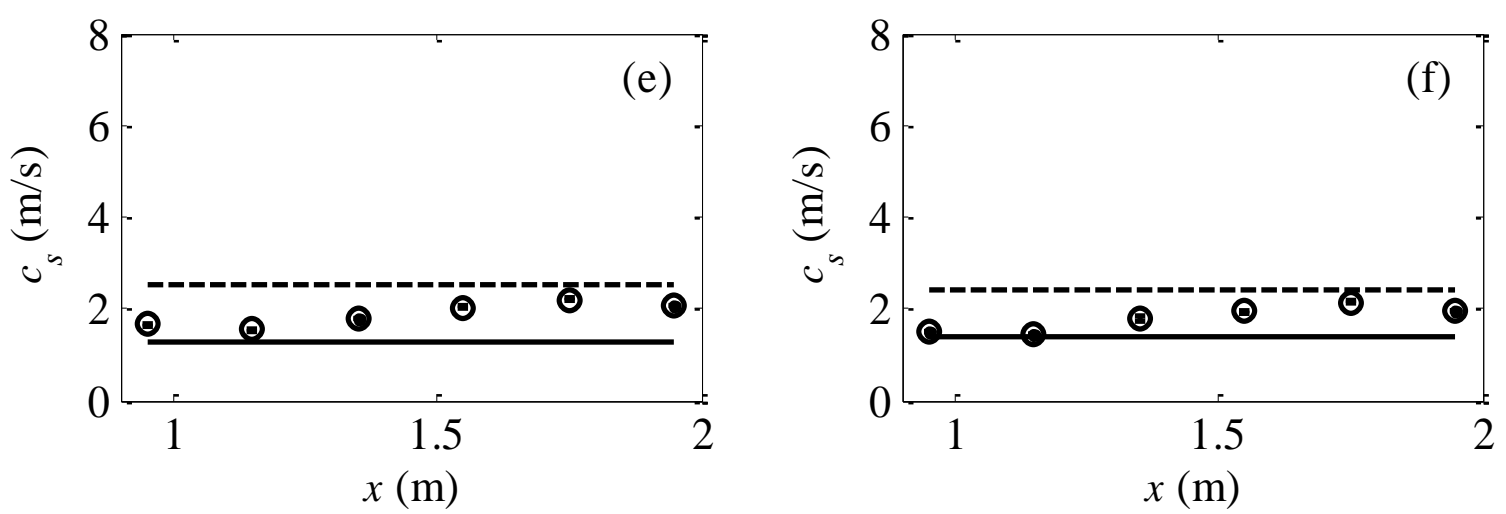

36 Figure 7: Wave celerity along the length of the long PP cover. Solid line = open water wave 37 celerity; dashed line $=$ model predictions (Wang and Shen, 2010) and circles $=$ experimental 38 data. $\mathrm{T}=$ (a) $0.5 \mathrm{~s}$, (b) $0.6 \mathrm{~s}$, (c) $0.7 \mathrm{~s}$, (d) $0.8 \mathrm{~s}$, (e) $0.9 \mathrm{~s}$ and, (f) $1.0 \mathrm{~s}$. 

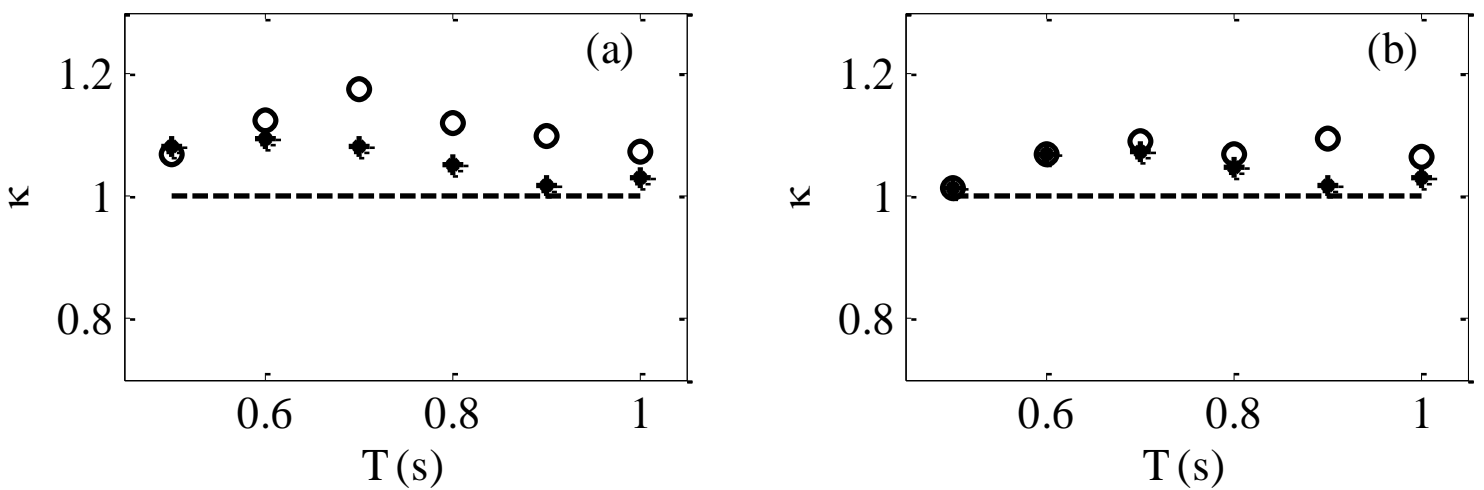

41
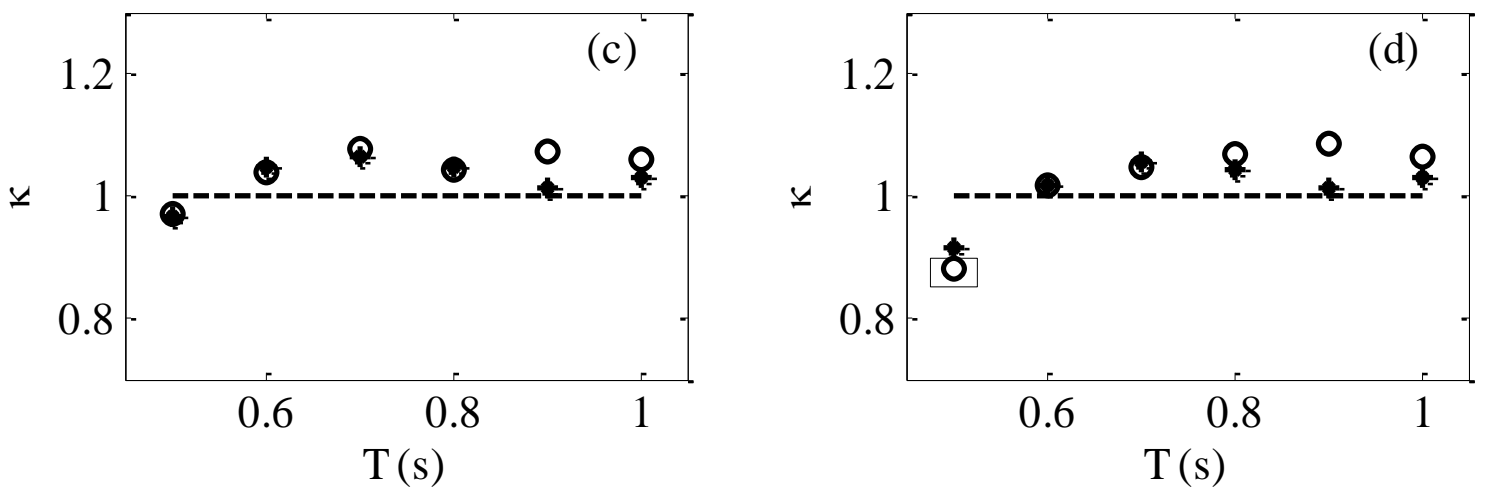

43

44 Figure 8: Normalized wave number under the PDMS cover against wave period. Dashed line = 45 open water case, circles $=$ experimental data, and asterisk $=$ model predictions $($ Wang and Shen, 46 2010). $m_{C A}=$ (a) $4 \%$, (b) $6 \%$, (c) $8 \%$ and, (d) $10 \%$. The symbol enclosed in a box corresponds to 47 the case when overwash was observed. 


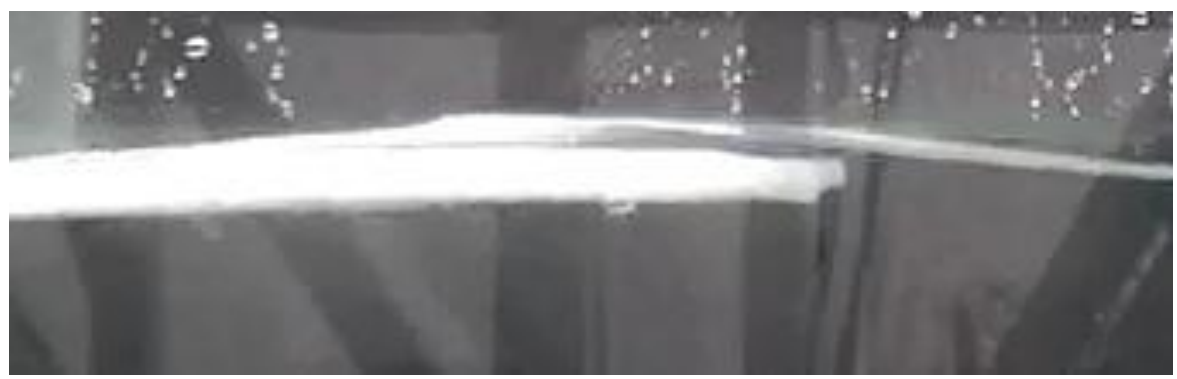

49

50

51 Figure 9: Over wash at the leading edge of the PDMS cover for the higher frequency case, $\mathrm{T}=$ 52 $0.5 \mathrm{~s}$ and $m_{C A}=10 \%$.

53 


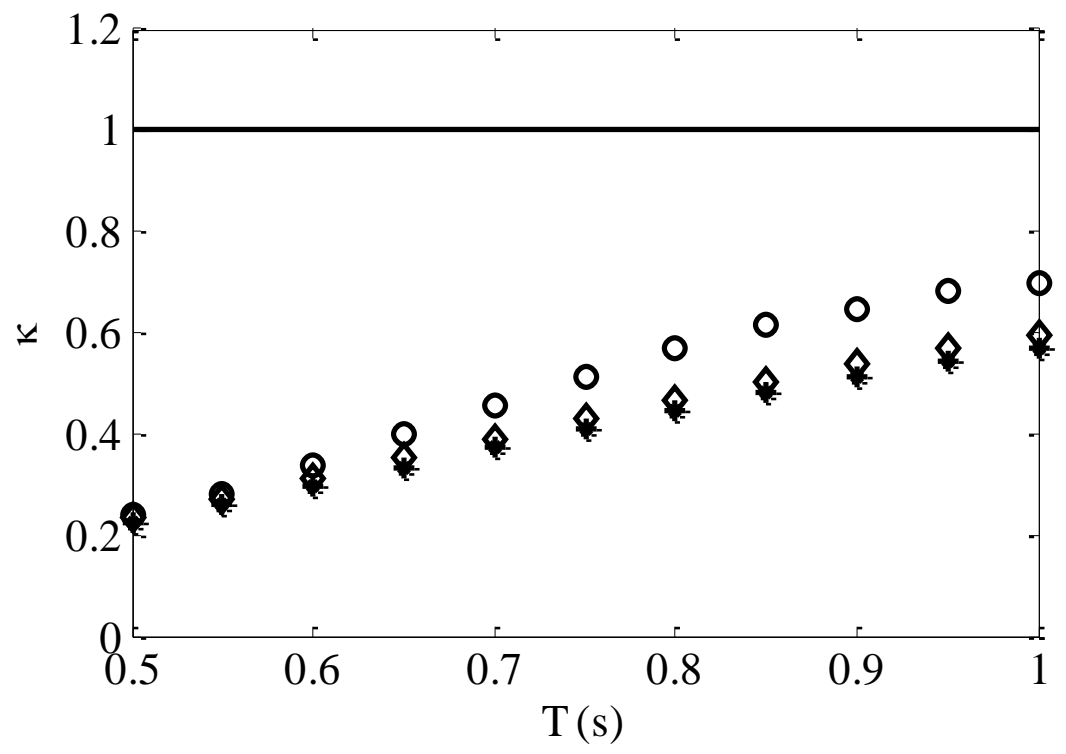

56 Figure 10: Normalized wave number under the $2.45 \mathrm{~m}$ long PP cover. Solid line = open water, 57 asterisk $=$ model predictions (Wang and Shen, 2010), diamonds $=$ Fox and Squire (1990) and 58 circles $=$ experimental data.

59

60 